\title{
An investigation of the longitudinal relationship between sleep and depressed mood in developing teens
}

This article was published in the following Dove Press journal:

Nature and Science of Sleep

13 February 2017

Number of times this article has been viewed

\section{Nicole Lovato' \\ Michelle A Short ${ }^{2}$ \\ Gorica Micic ${ }^{3}$ \\ Rachel M Hiller ${ }^{4}$ \\ Michael Gradisar ${ }^{3}$}

'Adelaide Institute for Sleep Health: A Flinders Centre of Research Excellence, Repatriation General Hospital, Flinders University, ${ }^{2}$ Centre for Sleep Research, University of South Australia, ${ }^{3}$ School of Psychology, Flinders University, Adelaide, SA, Australia; ${ }^{4}$ Department of Psychology, University of Bath, North East Somerset, UK
Correspondence: Nicole Lovato Adelaide Institute for Sleep Health: A Flinders Centre of Research Excellence, Repatriation General Hospital, Flinders University, GPO Box 2100, Adelaide, SA 500I, Australia

Tel +6I 882751990

Fax +61882776890

Email nicole.lovato@flinders.edu.au
Objective: The prospective, bidirectional relationship between sleep disturbance and depressed mood was assessed in a school-based sample of adolescents.

Method: One hundred and thirty-eight Australian adolescents (mean age time $1=15.69$, standard deviation $=0.92 ; 64 \%$ male) completed questionnaires to assess sleep parameters and depressed mood, on two occasions over 1 year.

Results: Cross-sectional associations were observed between depressed mood and sleep duration, as well as wakefulness in bed. Prospective analyses revealed depressed mood predicted less total sleep time on school nights and a longer latency to sleep onset on weekends 1 year later. There was no prospective support for sleep predicting later depressed mood.

Conclusion: Contrary to prediction, our results suggest in this case that depressed mood may act as a precursor to poor sleep rather than the converse.

Keywords: adolescence, sleep disturbance, school-based, prospective, depressive symptoms

\section{Introduction}

Approximately $25 \%$ of adolescents report symptoms of sleep disturbance, ${ }^{1}$ yet this rate can triple among those suffering depression (ie, up to $73 \%$ ). ${ }^{2}$ Although the association between sleep disturbance and depression among adolescents is well documented, ${ }^{3-9}$ the cross-sectional nature of this research means conclusions are limited regarding the developmental interplay between these two mental health problems. Only recently has the field begun to investigate the directional relationship between sleep disturbance and depression.

Using a meta-analytic technique, Lovato and Gradisar ${ }^{10}$ evaluated the strength of evidence for a directional relationship between sleep and depression in adolescents. Moderate effects were reported for various aspects of sleep being predictive of later depression (eg, latency to sleep onset, wakefulness after sleep onset [WASO], and the percent of sleep while in bed; overall effect sizes $=0.43-0.58, P<0.05$ ), yet a negligible effect of depression predicting later sleep disturbance (overall effect size $=-0.06$, $P>0.05)$. In particular, time spent awake in bed significantly contributed to the development of depression. ${ }^{10}$ Examination of effect sizes from longitudinal and treatment studies revealed at follow-up (mean $=5.64$ years) that adolescents with depression had significantly longer latencies to sleep onset (sleep onset latency [SOL]), more WASO and thus lower sleep efficiency (ie, the percent of sleep while in bed), when compared to adolescents who were nonclinical or had undergone remission since their baseline assessment. 
Based on their findings, Lovato and Gradisar ${ }^{10}$ provide a model for understanding the development of depression from initial sleep disturbance (Figure 1). Lovato and Gradisar ${ }^{10}$ proposed the developmental delay of the near 24-hour circadian system (and consequently the sleep period) observed around pubertal onset is likely to increase SOL, creating an opportunity to lie awake in bed. ${ }^{4,11}$ Lovato and Gradisar ${ }^{10}$ report, across all the analyses performed in their paper that wakefulness in bed (SOL, WASO, and sleep efficiency), was the most consistent indicator of current and future depression in adolescents. Furthermore, their paper highlights that wakefulness in bed is implied in some of the qualitative markers of sleep disturbance when self-report is used ${ }^{12,13}$ and may reflect that those who are depressed are more inclined to have negative responses in their self-reports. ${ }^{14}$ Based on their results, Lovato and Gradisar ${ }^{10}$ conclude that those adolescents who are depressed spend more time lying awake in quiet darkness. Spending time awake in bed in the absence of visual (ie, darkness) and auditory stimuli (ie, quiet) is conducive to the intrusion of automatic thoughts and dysfunctional cognitions regarding the consequences of sleeplessness. ${ }^{15-17}$ These thoughts are highly repetitive, intrusive, and ruminative, similar to those characteristic of depression. ${ }^{18,19}$ Wakefulness in bed is proposed to reinforce ruminative thinking styles and, in doing so, perpetuate fur-

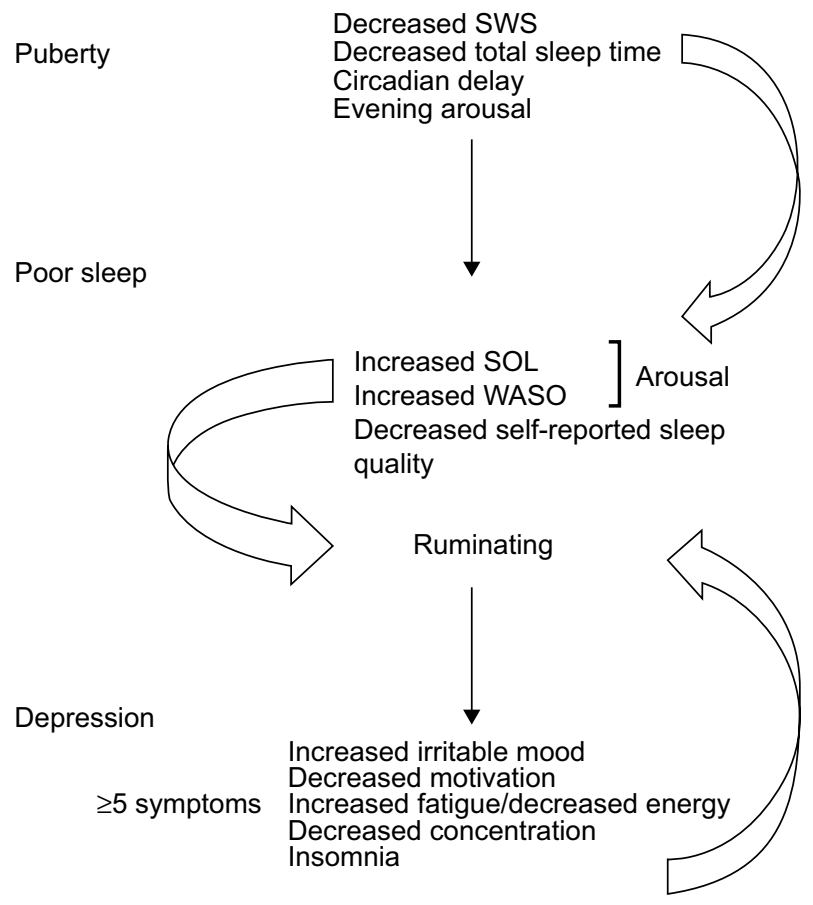

Figure I Conceptual model for the relationship between depression and sleep disturbance. Reproduced from Lovato N, Gradisar M. A meta-analysis and model of the relationship between sleep and depression in adolescents: recommendations for future research and clinical practice. Sleep Med Rev. 2014;18(6):52I-529. ${ }^{10}$

Abbreviations: SOL, sleep onset latency; WASO, wake after sleep onset; SWS, slow-wave sleep. ther sleep disturbance. ${ }^{20,21}$ Although these cognitive pathways are traditionally assumed to be unique to chronic insomnia, in their recent review, Richardson et $\mathrm{al}^{17}$ support the notion that the aforementioned sleep-disordered cognitive processes (intrusion of automatic thoughts, dysfunctional sleep-related beliefs) are also likely to play a critical role in maintaining delayed sleep timing, which is characteristic of many adolescents. Symptoms of depression and sleep disturbance overlap, including irritable mood, fatigue, reduced appetite (especially in the morning), and diminished ability to concentrate, ${ }^{12}$ and suggest that some symptoms may develop as a direct result of sleep disturbance, while others may do so indirectly (eg, fatigue may reduce adolescents' involvement in usual activities for which they subsequently lose interest). ${ }^{2,9,22,23}$

Lovato and Gradisar ${ }^{10}$ note the lack of prospective studies to confirm the findings of their meta-analysis and inform their proposed model. Although poor sleep quality and short sleep duration have both been demonstrated to prospectively predict poor subjective psychological well-being, ${ }^{24,25}$ few studies have applied this prospective methodology to investigate the directional relationship between sleep disturbance and depression per se. Roberts and Duong ${ }^{26}$ have provided the first steps toward achieving this goal. Their work examined the directional effect between sleep disturbance and depression in a large sample of 3,134 adolescents over a 1-year period. Their findings provide strong support for a bidirectional relationship between insomnia and depression. In their sample, insomnia increased the risk of major depression and depressive symptoms, while major depression, but not symptoms of depression per se, increased the risk of insomnia at follow-up. In a second study, this group ${ }^{27}$ replicated these findings, demonstrating a reciprocal relationship between sleep deprivation and depression. The current study aims to contribute to this developing body of published work ${ }^{26,27}$ by using a prospective research design to assess the directionality of this relationship.

The primary purpose of the current study is to confirm the findings of the meta-analysis by using a prospective design to assess the bidirectional relationship between sleep disturbance and depressed mood in a school-based sample of adolescents, and, in doing so, further inform the model proposed by Lovato and Gradisar. ${ }^{10}$ A strong association between sleep disturbance and depressed mood was predicted. Based on findings of a recent meta-analysis, ${ }^{10}$ it was predicted that wakefulness in bed (SOL, WASO) and reduced sleep duration (total sleep time) would prospectively predict depressed mood at 1-year follow-up, rather than conversely. 


\section{Methods}

\section{Participants and procedures}

Three hundred and forty-five adolescents in years 9, 10, and 11 years (age range $=13.5-18.9$ years; $60 \%$ male) were recruited from seven schools in Adelaide, South Australia. The schools were selected from a stratified sample based on socioeconomic status. During class time, adolescents completed the School Sleep Habits Survey (SSHS) with questions targeting lifestyle, health, and sleep variables ("Measures" section). ${ }^{28,29}$ Time 1 measures were part of a larger study that have been published elsewhere. ${ }^{30}$ Although we did not intend to conduct a follow-up assessment, the adolescents were contacted 1 year later and asked to complete the SSHS a second time. Of the 345 who provided data for time 1, 141 students (40.9\%) consented to participate 1 year later at time 2. Adolescents who could not be contacted or failed to consent at time 2 did not differ significantly from those who provided follow-up data on any demographic, sleep, or depressed mood variable at time 1 (all $P$ values $\geq 0.07$ ). Three adolescents were excluded from analyses given they did not provide data on depressed mood at time 2 . The final sample therefore comprised 138 adolescents. Data collection occurred from April 2008 to April 2010, during school terms, at least 2 weeks on either side of school holidays ${ }^{31}$ and not during daylight savings ${ }^{32}$ to avoid confounding influences on sleep. Consent was acquired from the adolescent and their legal guardian. Ethics approval was obtained from the Flinders University Social and Behavioural Research Ethics Committee and the Department of Education and Children's Services of South Australia for both assessment times.

\section{Measures}

Depressed mood was assessed using the Centre for Epidemiologic Studies Depression Scale (CES-D). ${ }^{33}$ Adolescents were presented with 20 statements and required to rate their agreement with each statement over the past week, using a four-point Likert scale ( $0=$ "rarely or none of the time, less than 1 day" and $3=$ "most or all of the time, 5-7 days"). The CES-D includes one sleep-related item "My sleep was restless", which was removed for the current analyses. Therefore, total scores range from 0 to 57 , with higher scores indicating greater depressed mood. The CES-D is valid and reliable for use in adolescents, displaying good concurrent validity, in addition to longitudinal and sex invariance.

Subjective sleep quality, including SOL (time taken to fall asleep; in minutes), total sleep time (in hours), and WASO (time spent awake during the night; in minutes), on school nights and weekends, was assessed using the SSHS. ${ }^{28,29}$ The
SSHS asks adolescents to report their sleep/wake behaviors over the past 2 weeks.

Sex (male coded as 1, female coded as 2) and age at time 1 were also used as potential predictors of depressed mood and sleep at time 2 .

\section{Statistical analyses}

All analyses were performed using SPSS for Windows, version 22 (IBM Corporation, Armonk, NY, USA). An $\alpha$ level of 0.05 was used to determine statistical significance. A series of between group one-way analysis of variances were conducted to assess potential differences in demographics, sleep, and depressed mood at time 1 and 2 . Table 1 shows the mean (and standard deviation) and within-subject effect size (Cohen's $d$ ) for each demographic, sleep, and depressed mood variable at both time 1 and 2 . Significant differences between these variables at time 1 and 2 are also denoted in Table 1 .

Associations between these demographic, sleep, and depressed mood variables at time 1 and 2 were assessed using correlational analyses. Age, sex, depressed mood, SOL, total sleep time, and WASO were correlated at both time 1 and 2 to demonstrate any cross-sectional relationships. Correlational analyses were also conducted between these variables from time 1 to 2 to provide an insight into potential prospective relationships. The correlation coefficient $(r)$ and significance value are shown for each of these analyses in Table 2.

Hierarchical multiple regression analyses were used to assess the predictive role of sleep for depressed mood and vice versa, both cross-sectionally at time 1 and prospectively from time 1 to 2 . The sleep-related outcome variables were SOL, WASO, and total sleep time. These outcome variables were considered separately for school nights and weekends.

Table I Mean values (and standard deviation) and withinsubject effect sizes for demographic, sleep, and depressed mood characteristics of the school-based sample

\begin{tabular}{|c|c|c|c|c|}
\hline $\begin{array}{l}\text { Predictor } \\
\text { variable }\end{array}$ & & Time I & Time 2 & $\begin{array}{l}\text { Within- } \\
\text { subject } \\
\text { effect sizes }^{a}\end{array}$ \\
\hline Age & & $15.69(0.92)$ & I $6.48(0.94)^{* *}$ & -0.85 \\
\hline Sex (male) & & $89(64 \%)$ & 89 (64\%) & 0.00 \\
\hline CES-D & & I I.88 (9.50) & I $8.44(13.94)^{* *}$ & -0.55 \\
\hline SOL & School & $34.35(31.48)$ & $27.81(27.98)^{* *}$ & 0.22 \\
\hline (minutes) & Weekend & $24.36(24.87)$ & I $7.82(16.54)^{* *}$ & 0.31 \\
\hline TST & School & $8.19(1.18)$ & $7.99(1.11)^{*}$ & 0.17 \\
\hline (hours) & Weekend & 9.35 (1.54) & $9.19(1.08)$ & 0.12 \\
\hline WASO & School & $9.76(22.15)$ & $|4.8|(32.85)$ & -0.18 \\
\hline (minutes) & Weekend & $9.74(20.64)$ & $19.83(37.56)$ & -0.33 \\
\hline
\end{tabular}

Notes: 'Effect sizes were calculated using Cohen's $d$. **, $\leq 0.00 \mathrm{I} ; *, \leq 0.05$. Abbreviations: CES-D, Centre for Epidemiologic Studies Depression Scale; SOL, sleep onset latency; TST, total sleep time; WASO, wake after sleep onset. 
Table 2 Correlations among demographic and predictor variables at time I and 2

\begin{tabular}{|c|c|c|c|c|c|c|c|c|c|c|}
\hline \multirow[t]{2}{*}{ Predictors } & & \multirow[t]{2}{*}{ Age } & \multirow[t]{2}{*}{ Sex } & \multicolumn{2}{|l|}{ SOL } & \multicolumn{2}{|l|}{ TST } & \multicolumn{2}{|l|}{ WASO } & \multirow[t]{2}{*}{ CES-D } \\
\hline & & & & School & Weekend & School & Weekend & School & Weekend & \\
\hline \multicolumn{11}{|c|}{ Predictors at time I } \\
\hline Age & & & -0.06 & -0.13 & -0.04 & $-0.22 * *$ & $-0.22 * *$ & 0.07 & 0.00 & 0.06 \\
\hline Sex & & -0.06 & & 0.05 & 0.03 & & 0.01 & -0.04 & 0.03 & 0.05 \\
\hline \multirow[t]{2}{*}{ SOL } & School & -0.13 & 0.05 & & $0.5 I^{* *}$ & $-0.4 I^{* *}$ & $-0.28 * *$ & 0.15 & 0.06 & 0.12 \\
\hline & Weekend & -0.05 & 0.03 & $0.5 I^{* *}$ & & $0.29 * *$ & -0.08 & 0.12 & -0.01 & $0.17 *$ \\
\hline \multirow[t]{2}{*}{ TST } & School & $0.22 *$ & -0.11 & $-0.4 I^{* *}$ & $-0.29 * *$ & & $0.35 * *$ & $-0.39 * *$ & -0.04 & $-0.36 * *$ \\
\hline & Weekend & $0.22 *$ & 0.01 & $-0.28 * *$ & -0.08 & $0.35 * *$ & & -0.09 & $-0.30 * *$ & 0.02 \\
\hline \multirow[t]{2}{*}{ WASO } & School & 0.07 & -0.04 & 0.15 & 0.12 & $0.39 * *$ & -0.09 & & $0.26 * *$ & $0.17^{*}$ \\
\hline & Weekend & 0.00 & 0.03 & 0.06 & -0.12 & -0.04 & $-0.30 * *$ & $0.26 * *$ & & 0.02 \\
\hline \multicolumn{2}{|l|}{ CES-D } & 0.06 & 0.05 & 0.12 & $0.17^{*}$ & $-0.36 * *$ & 0.02 & $0.17^{a}$ & 0.05 & \\
\hline \multicolumn{11}{|c|}{ Predictors at time $I$ and 2} \\
\hline \multicolumn{2}{|l|}{ Age (2) } & $0.90 * *$ & -0.11 & -0.13 & -0.08 & $-0.20 *$ & $-0.18 *$ & 0.08 & -0.01 & 0.05 \\
\hline \multicolumn{2}{|l|}{ Sex (2) } & -0.07 & $0.96 * *$ & 0.05 & 0.05 & -0.13 & 0.03 & -0.06 & 0.00 & 0.08 \\
\hline \multirow[t]{2}{*}{ SOL (2) } & School & -0.15 & 0.04 & $0.71 * *$ & $0.47 * *$ & $-0.25 * *$ & $-0.22 * *$ & 0.07 & 0.08 & 0.08 \\
\hline & Weekend & -0.13 & $0.19 *$ & $0.37 * *$ & $0.43 * *$ & -0.10 & -0.04 & 0.11 & 0.10 & 0.16 \\
\hline \multirow[t]{2}{*}{ TST (2) } & School & -0.15 & -0.03 & $-0.31 * *$ & $-0.18^{*}$ & $0.56 * *$ & $0.32 * *$ & -0.16 & -0.05 & $-0.23^{* *}$ \\
\hline & Weekend & -0.16 & 0.02 & -0.04 & -0.09 & 0.15 & $0.49 * *$ & -0.06 & -0.14 & -0.07 \\
\hline \multirow[t]{2}{*}{ WASO (2) } & School & 0.09 & 0.03 & 0.05 & -0.03 & -0.06 & -0.08 & 0.12 & 0.10 & -0.07 \\
\hline & Weekend & 0.10 & -0.10 & -0.07 & -0.04 & 0.01 & -0.03 & -0.01 & -0.02 & -0.00 \\
\hline CES-D (2) & & 0.01 & 0.12 & 0.13 & 0.16 & $0.29 * *$ & 0.02 & 0.16 & 0.07 & $0.49 * *$ \\
\hline
\end{tabular}

Notes: (2) indicates time 2. **P $\leq 0.001 ; * P 0.05 ;{ }^{a} 0.05$

Abbreviations: CES-D, Centre for Epidemiologic Studies Depression Scale; SOL, sleep onset latency; TST, total sleep time; WASO, wake after sleep onset.

Table 3 Sleep variables at time I that predict depressed mood at time I and 2.

\begin{tabular}{llll}
\hline Predictors at time I & CES-D (Time I) & CES-D (Time 2) \\
\cline { 3 - 4 } & B & B \\
\hline Age & 0.01 & -0.02 \\
Sex & -0.01 & 0.08 \\
CES-D & & - & $0.42^{* *}$ \\
SOL & School & -0.06 & 0.002 \\
& Weekend & 0.10 & 0.04 \\
TST & School & $-0.42^{* *}$ & -0.13 \\
& Weekend & $0.19^{*}$ & 0.08 \\
WASO & School & -0.10 & 0.04 \\
& Weekend & 0.10 & 0.05 \\
\hline
\end{tabular}

Notes: ${ }^{*} B$ value is significant at the 0.05 level (two-tailed). ${ }^{* *} \beta$-value is significant at the 0.01 level (two-tailed).

Abbreviations: CES-D, Centre for Epidemiologic Studies Depression Scale; SOL, sleep onset latency; TST, total sleep time; WASO, wake after sleep onset.

The outcome variable for depressed mood was the total score on the CES-D (the total score obtained minus the single sleep-related item from the CES-D).

For analyses identifying sleep variables, which predict depressed mood at time 1 and 2, individual regression equations were conducted for each sleep variable, including SOL, total sleep time, and WASO. In each case, depressed mood (either at time 1 or 2) was entered as the outcome variable, while age and sex were entered as covariates. Depressed mood at time 1 was also added as a covariate for analyses used to identify predictors of depressed mood at time 2 . The $\beta$-value and significance for each regression equation are summarized in Table 3.
When assessing the predictive role of depressed mood for sleep, SOL, total sleep time, or WASO were considered as outcome variables. As previously described, individual regression equations were conducted for each sleep outcome variable (either at time 1 or 2) with depressed mood at time 1 entered as the predictor variable. Again, age and sex were entered as covariates. Table 4 shows the $\beta$-value and significance for each regression equation.

\section{Results}

Table 1 summarizes the demographic, sleep, and depressed mood characteristics of the sample. At time 2, the adolescents reported significantly greater depressed mood, shorter SOLs, and less total sleep time on school nights when compared to time 1 .

\section{Correlations among predictor variables}

Significant cross-sectional and prospective correlations between demographic variables, sleep, and depressed mood at time 1 and 2 are shown in Table 2. Significant cross-sectional correlations at time 1 were observed between depressed mood with SOL (small effect) on weekends, total sleep time (moderate effect) on school nights as well as WASO (small effect) on school nights. ${ }^{33}$ Cross-sectionally, at time 2, a nearmoderate correlation between school-night total sleep time and depressed mood held, however, there was no significant 
Table 4 Predictors of sleep at time I and 2.

\begin{tabular}{|c|c|c|c|c|c|c|c|c|c|c|c|c|}
\hline \multirow[t]{2}{*}{ Predictors } & \multicolumn{2}{|c|}{ SOL (Time I) } & \multicolumn{2}{|c|}{ TST (Time I) } & \multicolumn{2}{|c|}{ WASO (Time I) } & \multicolumn{2}{|c|}{ SOL (Time 2) } & \multicolumn{2}{|c|}{ TST (Time 2) } & \multicolumn{2}{|c|}{ WASO (Time 2) } \\
\hline & School & Weekend & School & Weekend & School & Weekend & School & Weekend & School & Weekend & School & Weekend \\
\hline & B & B & B & B & B & B & B & B & B & B & B & B \\
\hline Age & -0.14 & -0.06 & $-0.21 * *$ & $-0.22 * *$ & 0.06 & 0.00 & -0.15 & -0.13 & -0.14 & -0.15 & 0.10 & 0.09 \\
\hline Sex & 0.04 & 0.02 & $-0.34^{* *}$ & -0.00 & -0.05 & 0.02 & 0.03 & $0.18^{*}$ & -0.03 & 0.10 & 0.04 & -0.09 \\
\hline CES-D (I) & 0.12 & $0.17^{*}$ & -0.11 & 0.03 & $0.16+$ & 0.05 & 0.09 & $0.16+$ & $-0.22^{*}$ & -0.07 & -0.08 & 0.01 \\
\hline
\end{tabular}

Notes: $* B$ value is significant at the 0.05 level (two-tailed). ${ }^{* * B} B$ value is significant at the 0.01 level (two-tailed). ${ }^{+} B$ value is significant at $<0.07$.

Abbreviations: CES-D, Centre for Epidemiologic Studies Depression Scale; SOL, sleep onset latency; TST, total sleep time; WASO, wake after sleep onset.

correlation between school-night WASO and depressed mood. No significant prospective correlations between time 1 sleep variables and time 2 depressed mood were evident.

\section{The predictive role of sleep in the development of depressed mood}

The multivariate relationships between depressed mood, demographic, and sleep variables at time 1 and 2 are shown in Table 3. Hierarchical multiple regression analyses showed that time 1 total sleep time on both school nights and weekends were significant independent cross-sectional predictor of depressed mood at time 1. After controlling for age and sex, less total sleep time on school nights, and more total sleep time on weekends were predictive of greater depressed mood. However, total sleep time at time 1 did not significantly predict depressed mood prospectively at time 2 .

\section{The predictive role of depressed mood for sleep}

The multivariate relationships between depressed mood, demographic, and sleep variables at time 1 and 2 are shown in Table 4. Hierarchical multiple regression analyses revealed that time 1 depressed mood was a significant independent predictor of SOL on weekends at time 1, after controlling for age and sex. Although not statistically significant, depressed mood is somewhat predictive of WASO on school nights. The predictive value of depressed mood for sleep was also observed prospectively. At time 2, depressed mood was a significant predictor of total sleep time on school nights, independent of age, sex, and depressed mood at time 1 . Depressed mood also remained somewhat predictive of SOL on weekends at time 2. In summary, greater depressed mood at time 1 significantly predicted a longer SOL on weekends at time 1, and less total sleep time on school nights at time 2. To a lesser extent, depressed mood also predicted more WASO on school nights at time 1 and a longer SOL on weekends at time 2 .

\section{Discussion}

The current study tested Lovato and Gradisar's ${ }^{10}$ metaanalysis, and proposed model of the relationship between sleep (ie, SOL, total sleep time, WASO) and depressed mood, in a sample of adolescents. Our data provide mixed support. Bidirectional cross-sectional relationships exist between sleep and depressed mood, yet prospective analyses provide limited support for the notion that wakefulness in bed acts as a precursor to the development of depression. Cross-sectional analyses revealed that less total sleep time on school nights and more total sleep time on weekends were associated with depressed mood at time 1 among this group of adolescents. This association was also reflected in our prospective analyses. Depressed mood at time 1 significantly predicted less total sleep time on school nights 1-year later at time 2. Although significant cross-sectional relationships between wakefulness in bed and depressed mood were observed (depressed mood was associated with longer latency to sleep onset and more WASO), there was no prospective support for sleep predicting later depressed mood. Instead, and contrary to the recent meta-analysis, ${ }^{10}$ depressed mood at time 1 predicted wakefulness in bed (longer latency to sleep onset) at time 2 .

In contrast to earlier research, the current findings do not support the notion that sleep disturbance acts as a precursor for depressed mood. There are several methodological factors, which may explain the disparity between the current findings and earlier work. Previous studies, ${ }^{34-40}$ including those that form the basis of the recent meta-analysis, ${ }^{10}$ which have assessed the predictive role of sleep disturbance in the development of depressed mood have defined sleep disturbance objectively (polysomnography) in adolescents diagnosed major depressive disorder. This is quite distinct from the methodology of the present investigation that defined sleep in subjective terms via self-report in a nonclinical, school-based sample of adolescents with depressed mood. It is important to recognize the directional relationship between 
depressed mood and self-reported sleep disturbance may be quite distinct from those relationships operating between major depressive disorder and objectively determined sleep disturbance. This remains an empirical question and is recommended as the subject of further investigations in this field.

Although several studies have assessed the association between sleep and depression, ${ }^{3,4,6-8,41-43}$ the notion that depressed mood may act as a precursor to the development of sleep disturbance remains largely uninformed. The current study is one of only three studies that have assessed the reciprocal prospective relationship between depressed mood and sleep, using the same sample of adolescents. ${ }^{26,27}$ Roberts and Duong found that both self-reported sleep deprivation ( $\leq 6$ hours of sleep per night over a 4 -week period $)^{27}$ and symptoms of insomnia ${ }^{26}$ were reciprocally related to major depression over a 1-year period in a community-based cohort of adolescents. Adolescents were categorized as suffering from insomnia irrespective of whether they reported trouble falling asleep, frequent awakenings during the night, waking up too early, or experienced nonrestorative sleep retrospectively over a 4 -week period. The present study was not able to replicate these findings using self-reported quantification of sleep. Future research is encouraged to measure both adolescents' perception of sleep quality and insomnia (ie, difficulties initiating/maintaining sleep), as well as sleep parameters (ie, SOL, WASO) to provide a clear indication regarding which aspect(s) of sleep disturbance are related to major depression.

\section{Theoretical and clinical implications}

The current prospective analyses provide mixed support for the model developed by Lovato and Gradisar ${ }^{10}$ whereby the development of depression is proposed to arise from initial sleep disturbance. More specifically, Lovato and Gradisar ${ }^{10}$ propose wakefulness in bed acts to reinforce ruminative thinking styles and, in doing so, perpetuates further sleep disturbance. Their meta-analysis findings suggest these processes could develop into depression over time. This new prospective data, taken together with earlier studies, ${ }^{26,27}$ suggests that depressed mood may also serve to perpetuate wakefulness in bed. The mechanism for this process remains speculative. It is proposed that teens with depressed mood may spend more time in bed, which, over time, contributes to less total sleep time and increased time spent awake in bed. Future research is encouraged to investigate possible mechanisms underpinning this relationship.
The current findings continue to support the importance of establishing and maintaining good sleep during adolescence using cognitive-behavioral approaches. ${ }^{10}$ Cognitive restructuring can target cognitions and ruminative thinking, akin to depression, while attempting to fall asleep, and during bouts of wakefulness across the night. ${ }^{22}$ It is recommended that these cognitive techniques are used in combination with behavioral techniques to reduce time spent awake in bed (ie, preferably stimulus control therapy over sleep restriction therapy in order to extend sleep quantity) to target the perpetuation of the sleep disturbance.

\section{Limitations}

Although a 1-year period is not ideal to prospectively assess bidirectional relationships, the current data and the previous work of Roberts and Duong ${ }^{26,27}$ nevertheless indicate directional relationships between depressed mood and wakefulness in bed over this brief period may occur. In fact, the time course of the effects of insufficient and/or poor sleep may be relatively short. Perhaps part of the issue is that the effect has already occurred and what is really being assessed is those few cases where chronicity is a problem. For other adolescents, these relationships may have already been resolved and thus, these effects are not reflected in this type of data. Future research should endeavor to extend this assessment period over several years to incorporate younger ages at baseline (mean age of the current sample at time $1=15$ years) and longer follow-ups, while maintaining frequent sampling. Although prospective studies are practically more difficult to perform, replicating them, and disseminating their findings, even if no effects are found, are important issues in this field. ${ }^{12,44,45}$

It is important to acknowledge that the use of selfreported sleep data may not be viewed as ideal. However, actigraphy estimates of sleep have shown more WASO and less total sleep time, especially among adolescent boys. ${ }^{46}$ Therefore, such objective measures do not necessarily come without flaws. Although the effects of age, sex, and time 1 depressed mood were controlled for in the current analysis, several potentially confounding variables (eg, anxiety) and the role of protective factors (eg, physical activity, parentchild relationship) could not be used. ${ }^{47-49}$ These constitute important avenues for future research. Psychiatric interviews were not conducted with the adolescents involved in this study. Given $50 \%$ of psychiatric disorders emerge before the age of 15 years, ${ }^{50}$ assessment of the directional relationship between sleep disturbance and psychiatric symptoms 
in these adolescents would be equally valuable in this field of research.

\section{Conclusion}

The current study assessed the prospective, bidirectional relationship between sleep disturbance and depressed mood in a school-based sample of adolescents. Our data continue to support the association between wakefulness in bed and depressed mood. Moreover, our data suggest depressed mood may serve to perpetuate wakefulness in bed and reduce total sleep time. These findings highlight the importance of establishing and maintaining good sleep during adolescence. More comprehensive studies assessing the prospective, reciprocal relationship between sleep disturbance and depression are required to inform the developmental interplay these two mental health problems. Future research is encouraged to examine developmental trajectories of these problems from childhood through adolescence into adulthood. ${ }^{33}$

\section{Disclosure}

The authors report no conflicts of interest in this work.

\section{References}

1. McKnight-Eily LR, Eaton DK, Lowry R, Croft JB, Presley-Cantrell L, Perry GS. Relationships between hours of sleep and health-risk behaviors in US adolescent students. Prev Med. 2011;53(4-5):271-273.

2. Lui X, Buysse DJ, Gentzler AL, et al. Insomnia and hypersomnia associated with phenomenology and comorbidity in childhood depression. Sleep. 2007;30(1):83-90.

3. 3.Appelboom-Fondu J, Kerkhofs M, Mendlewicz J. Depression in adolescents and young adults-polysomnographic and neuroendocrine aspects. J Affect Disord. 1988;14(1):35-40.

4. Bennett DS, Ambrosini PJ, Kudes D, Metz C, Rabinovich H. Gender differences in adolescent depression: do symptoms differ for boys and girls? J Affect Disord. 2005;89(1-3):35-44.

5. Forbes EE, Bertocci MA, Gregory AM, Ryan ND, Axelson DA, Birmaher B, Dahl RE. Objective sleep in pediatric anxiety disorders and major depressive disorder. $\mathrm{J} \mathrm{Am} \mathrm{Acad} \mathrm{Child} \mathrm{Adolesc} \mathrm{Psychiatry.}$ 2008;47(2):148-155.

6. Patton GC, Coffey C, Posterino M, Carlin JB, Wolfe R. Adolescent depressive disorder: a population-based study of ICD-10 symptoms. Aust N Z J Psychiatry. 2000;34(5):741-747.

7. Riemann D, Kammerer J, Low H, Schmidt MH. Sleep in adolescents with primary major depression and schizophrenia: a pilot study. JChild Psychol Psychiatry. 1995;36(2):313-326.

8. Roane BM, Taylor DJ. Adolescent insomnia as a risk factor for early adult depression and substance abuse. Sleep. 2008;31(10):1351-1356.

9. Wiebe ST, Cassoff J, Gruber R. Sleep patterns and the risk for unipolar depression: a review. Nat Sci Sleep. 2012;4:63-71.

10. Lovato N, Gradisar M. A meta-analysis and model of the relationship between sleep and depression in adolescents: recommendations for future research and clinical practice. Sleep Med Rev. 2014;18(6): $521-529$.

11. Maalouf FT, Brent DA. Child and adolescent depression intervention overview: what works, for whom and how well? Child Adolesc Psychiatr Clin N Am. 2012;21(2):299-312.

12. American Psychiatric Association. Diagnostic and Statistical Manual of Mental Disorders Test Revision. 4th ed. Washington, DC: American Psychiatric Association; 2001
13. Gibson ES, Powles AC, Thabane L, et al. "Sleepiness" is serious in adolescence: two surveys of 3235 Canadian students. BMC Public Health. 2006;6:116.

14. Beck AT. Depression: Clinical, Experimental and Theoretical Aspects. New York: International Universities Press: Harper \& Row; 1967.

15. Danielsson NS, Harvey AG, Macdonald S, Jansson-Fröjmark M, Linton SJ. Sleep disturbance and depressive symptoms in adolescence: the role of catastrophic worry. J Youth Adolesc. 2013;42(8):1223-1233.

16. Hiller R, Lovato N, Gradisar M, Oliver M, Slater A. Trying to fall asleep whilst catastrophising: What sleep-disordered adolescents think and feel. Sleep Med. 2013;15(1):96-103.

17. Richardson C, Gradisar M, Barbero SC. Are cognitive "insomnia" processes involved in the development and maintenance of delayed sleep wake disorder? Sleep Med Rev. 2016;26:1-8.

18. Abela JRZ, Vanderbilt E, Rochon A. A test of the integration of the response styles and social support theories of depression in third and seventh grade children. J Soc Clin Psychol. 2004;23(5):653-674.

19. Papadakis A, Prince R, Jones N, Strauman T. Self-regulation, rumination, and vulnerability to depression in adolescent girls. Dev Psychopathol. 2006;18(3):815-829.

20. Gregory AM, Cox J, Crawford MR, Holland J, Harvey AG. Dysfunctional beliefs and attitudes about sleep in children. J Sleep Res. 2009; 18(4):422-426.

21. Wicklow A, Espie CA. Intrusive thoughts and their relationship to actigraphic measurement of sleep: towards a cognitive model of insomnia. Behav Res Ther. 2000;38(7):679-693.

22. Clarke G, Harvey AG. The complex role of sleep in adolescent depression. Child Adolesc Psychiatr Clin N Am. 2012;21(12):385-400.

23. Ohayon MM, Shapiro CM, Kennedy SH. Differentiating DSM-IV anxiety and depressive disorders in the general population: comorbidity and treatment consequences. Can J Psychiatry. 2000;45(2): 166-172.

24. Brand S, Hatzinger M, Stadler C, et al. Does objectively assessed sleep at five years predict sleep and psychological functioning at 14 years? Hmm, yes and no! J Psychiatr Res. 2015;60:148-155.

25. Kalak N, Gerber M, Kirov R, Mikoteit T, Pühse U, Holsboer-Trachsler $\mathrm{E}$, Brand S. The relation of objective sleep patterns, depressive symptoms, and sleep disturbances in adolescent children and their parents: a sleep-EEG study with 47 families. $J$ Psychiatr Res. 2012;46(10): 1374-1382.

26. Roberts RE, Duong HT. Depression and insomnia among adolescents: A prospective perspective. J Affect Disord. 2013;148(1):66-71.

27. Roberts RE, Duong HT. The prospective association between sleep deprivation and depression among adolescents. Sleep. 2014;37(2): 239-244.

28. Wolfson AR, Carskadon MA, Acebo C, Seifer R, Fallone G, Labyak SE, Martin JL. Evidence for the validity of a sleep habits survey for adolescents. Sleep. 2003;26:213-216.

29. Wolfson AR, Carskadon MA. (1998). Sleep schedules and daytime functioning in adolescents. Child Dev. 1998;69(4):875-887.

30. Short MA, Gradisar M, Lack LC, Wright H, Dohnt H. The sleep patterns and well-being of Australian adolescents. J Adolesc. 2013;36(1):103-110.

31. Warner S, Murray G, Meyer D. Holiday and school-term sleep patterns of Australian adolescents. J Adolesc. 2008;31(5):595-608.

32. Schneider A, Randler C. Daytime sleepiness during transition into daylight saving time in adolescents: Are owls higher at risk? Sleep Med. 2009;10(9):1047-1050.

33. Radloff LS. The CES-D Scale: A self-report depression scale for research in the general population. Applied Psychol Meas. 1977;1(3): 385-401.

34. Goetz RR, Wolk SI, Coplan JD, Ryan ND, Weissman MM. Premorbid polysomnographic signs in depressed adolescents: a reanalysis of EEG sleep after longitudinal follow-up in adulthood. Biol Psychiatry. 2001;49(11):930-942.

35. McCracken JT, Poland RE, Lutchmansingh P, Edwards C. Sleep electroencephalographic abnormalities in adolescent depressives: effects of scopolamine. Biol Psychiatry. 1997;42(7):577-584. 
36. Rao U, Dahl RE, Ryan ND, Birmaher B, Williamson DE, Rao R, Kaufman J. Heterogeneity in EEG sleep findings in adolescent depression: unipolar versus bipolar clinical course. $J$ Affect Disord. 2002;70(3):273-280.

37. Rao U, Dahl RE, Ryan ND, et al. The relationship between longitudinal clinical course and sleep and cortisol changes in adolescent depression. Biol Psychiatry. 1996;40(6):474-484.

38. Rao U, McCracken JT, Lutchmansingh P, Edwards C, Poland RE. Electroencephalographic sleep and urinary free cortisol in adolescent depression: a preliminary report of changes from episode to recovery. Biol Psychiatry. 1997;41(3):369-373.

39. Rao U, Poland RE. Electroencephalographic sleep and hypothalamicpituitary-adrenal changes from episode to recovery in depressed adolescents. J Child Adolesc Psychopharmacol. 2008;18(6):607-613.

40. Shain BN, Naylor M, Shipley JE, Alessi N. Imipramine effects on sleep in depressed adolescents: a preliminary report. Biol Psychiatry. 1990;28(5):459-462.

41. Dahl RE, Puig-Antich J, Ryan ND, et al. EEG sleep in adolescents with major depression: the role of suicidality and inpatient status. $J$ Affect Disord. 1990;19(1):63-75.

42. Khan AU, Todd S. Polysomnographic findings in adolescents with major depression. Psychiatry Res. 1990;33(3):313-320.
43. Park HY, Heo J, Subramanian SV, Kawachi I, Oh J. Socioeconomic inequalities in adolescent depression in South Korea: a multilevel analysis. PLoS One. 2012;7(10):e47025.

44. Rosenthal R. Replication in behavioral research. J Soc Behav Pers. 1990;54(4):1-30.

45. Schmidt S. Shall we really do it again? The powerful concept of replication is neglected in the social sciences. Rev Gen Psychol. 2009;13(2):90-100.

46. Short MA, Gradisar M, Lack LC, Wright H, Carskadon MA. The discrepancy between actigraphic and sleep diary measures of sleep in adolescents. Sleep Med. 2012;13(4):378-384.

47. Gangwisch JE, Babiss LA, Malaspina D, Turner JB, Zammit GK, Posner $\mathrm{K}$. Earlier parental set bedtimes as a protective factor against depression and suicidal ideation. Sleep. 2010;33(1):97-106.

48. Kalak N, Lemola S, Brand S, Holsboer-Trachsler E, Grob, A. Sleep duration and subjective psychological well-being in adolescence: a longitudinal study in Switzerland and Norway. Neuropsychiatr Dis Treat. 2014; 10:1199-1207.

49. Sigfusdottir ID, Asgeirsdottir BB, Sigurdsson JF, Gudjonsson GH. Physical activity buffers the effects of family conflict on depressed mood: a study on adolescent girls and boys. J Adolesc. 2011;34(5):895-902.

50. Paus T, Keshavan M, Giedd J. Why do many psychiatric disorders emerge during adolescence? Nat Rev Neurosci. 2008;9(12):947-957.
Nature and Science of Sleep

\section{Publish your work in this journal}

Nature and Science of Sleep is an international, peer-reviewed, open access journal covering all aspects of sleep science and sleep medicine, including the neurophysiology and functions of sleep, the genetics of sleep, sleep and society, biological rhythms, dreaming, sleep disorders and therapy, and strategies to optimize healthy sleep. The manuscript

\section{Dovepress}

management system is completely online and includes a very quick and fair peer-review system, which is all easy to use. Visit http://www. dovepress.com/testimonials.php to read real quotes from published authors. 